\title{
Conjecturing via reconceived classical analogy
}

\author{
Kyeong-Hwa Lee $\cdot$ Bharath Sriraman
}

Published online: 9 October 2010

(C) The Author(s) 2010. This article is published with open access at Springerlink.com

\begin{abstract}
Analogical reasoning is believed to be an efficient means of problem solving and construction of knowledge during the search for and the analysis of new mathematical objects. However, there is growing concern that despite everyday usage, learners are unable to transfer analogical reasoning to learning situations. This study aims at facilitating analogy use for conjecturing in discourse-rich mathematics classrooms. We reconceptualized one of the traditional perspectives on analogical reasoning, called classical analogy, as a more dynamic one by providing learners with the opportunity to choose a target object and its property. While shifting attention to particular aspects of mathematical activity, we observed and analyzed how students became aware of hidden relational similarities and utilized them while weakening others to make conjectures. The detailed analysis of the constructs and processes of several similarity-making and conjecturing activities supports the significance of reconceived classical analogy use in mathematics classrooms.
\end{abstract}

Keywords Analogical reasoning $\cdot$ Knowledge construction $\cdot$ Relational similarity Conjecturing · Justifying

\section{Introduction}

Analogical reasoning, in general, refers to the ability to perceive and construct corresponding structural similarity in objects whose surface features are not necessarily similar (Richland, Holyoak \& Stigler, 2004, p. 37). Research has shown that children can use analogical reasoning to adapt to new novel contexts (Holyoak \& Thagard, 1989), transfer representations across contexts (Novick, 1988; White \& Mitchelmore, 2010),

K.-H. Lee $(\bowtie)$

Department of Mathematics Education, Seoul National University, Shillim-dong, Kwanak-gu, Seoul 151-748, Korea

e-mail:khmath@snu.ac.kr

B. Sriraman

The University of Montana, Missoula, MT, USA

e-mail: sriramanb@mso.umt.edu 
understand and solve word problems (Reed, Dempster \& Ettinger, 1985; Bassok, 2001), and solve comparison problems (English, 1997). However, unlike science education scant attention has been given in mathematics education to analogical reasoning as a conceptdevelopment skill (English \& Sharry, 1996, p. 138).

Knowledge construction or concept development in mathematics education has been described as repeated abstraction (Boero, Dreyfus, Gravemeijer, Gray, Hershkowitz, Schwarz, Sierpinska \& Tall, 2002). Analogical reasoning plays an important role in the process of abstraction through investigating similarities and discerning structures (Sriraman, 2004). However, there is growing concern that despite everyday usage, learners are unable to transfer analogical reasoning to learning situations (Dunbar, 2001; Lobato, Ellis \& Muñoz, 2003; Leech, Mareschal \& Cooper, 2008). Thus, mathematics education would benefit greatly from studies on the use of analogical reasoning as an instructional device for conjecturing in discourse-rich mathematics classrooms. Our present study fills in some of the missing gaps in the existing line of research on analogical thinking.

\section{Conceptual framework}

In this section, we synthesize the work of major theorists who have examined reasoning and conjecturing via analogies, as well as those who have investigated abstraction processes in mathematical thinking and learning. In doing so, we draw on the works of post-Piagetian researchers (e.g., Dubinsky, 1991; Gray \& Tall, 1994) as well as those using a more sociocultural and contextual approach towards abstraction (e.g., Hershkowitz, Hadas, Dreyfus \& Schwarz, 2007; White \& Mitchelmore, 2010). In post-Piagetian frameworks, reification is the dominant mode of explaining the process of abstraction, in which processes become objects of manipulation in their own right. This view is an extension of Skemp's terminology by Gray and Tall (1994). In socio-cultural and contextual approaches, shifts in conceptualizing that occur in discourse-rich environments, via metaphors and reflective discourse in the classroom facilitate reflective abstraction (Cappetta \& Zollman, 2009; Hershkowitz et al., 2007). Although there is an inherent tension in these two accounts of abstraction, one that is logico-deductive, and the other socio-discursive, the two can be viewed as components of theories allowing for the possibility of complementary themes (Radford, 2008) which in turn can be made to network and provide different meanings, connections, or explanations to the phenomenon under investigation. Our conceptual framework consists of post-Piagetian and contextual/socio-cultural views of analogical thinking and abstraction, and in particular we use elements from theories of abstraction and reasoning by analogy to develop analogy problem solving as an instructional device for use in the classroom.

\subsection{Conjecturing by analogies}

Polya (1954) emphasized the value of conjecturing via analogies in mathematics learning, particularly how mathematicians utilized analogies when discovering new concepts or new problem solving methods. Numerous examples abound in the history of mathematics of mathematicians like Euler, Newton, and others taking "daring" steps, i.e., reasoning by induction or analogy, such as Newton's formula for binomial expansions with rational powers, and Euler's astonishing closed sum of the infinite series $1+1 / 4+1 / 9+$ $1 / 16+\ldots+1 / \mathrm{n}^{2}+\ldots$, in which he applied finite methods to infinite cases. However, Euler confidently guessed the sum of the series to be $\pi^{2} / 6$, did not attribute this coincidence 
to chance and boldly conjectured that the sum of this series was indeed $\pi^{2} / 6$, not to mention the fact that he later proved his conjecture to be true (Polya, 1954, pp.95-96).

Lakatos (1976) highlighted how conjecturing by analogies can contribute to mathematical discovery and some case studies utilizing a Lakatosian framework (Sriraman, 2006) indicate that conjecturing by analogies occurs in mathematics classrooms although this is more an exception than the rule. For instance in empirical studies based on observation of mathematics classrooms, Richland et al. (2004, p. 55) found only $2 \%$ of analogies were produced by students. As the researchers interpreted, "teachers may be failing to provide an important learning opportunity for students by maintaining control over the reasoning process" (Richland et al., p. 58). This warrants the necessity of research on facilitating analogy use in mathematics classrooms.

\subsection{Re-conception of classical analogy}

There are three types of analogies that have been used in mathematics education: classical analogy, problem analogy, and pedagogical analogy (English, 2004). ${ }^{1}$ Among the three aforementioned types of analogies, problem analogy and pedagogical analogy were widely used as heuristics in mathematics learning. However, classical analogy problems are mainly applied measurements of intelligence and reasoning ability development exercises, rather than used as a domain-specific cognitive skill in mathematics learning. Whereas previous research on analogical reasoning in mathematics education has focused much on the use of problem analogy and pedagogical analogy, few researches have considered analogical reasoning in a more broad sense (e.g., English \& Sharry, 1996; Zaslavsky, 2008; Lee, 2009; Cañadas, Deulofeu, Figueiras, Reid \& Yevdokimov, 2007, etc.). As a result of this instructional approach, learners were able to voice diverse views on relational similarities. One new perspective on the transfer of learning called actor-oriented transfer (Lobato \& Siebert, 2002), supports the use of analogical reasoning in a broader sense rather than just the application of the aforementioned three analogies. Based on empirical evidence, Lobato and Siebert claim that a teacher should develop better understanding of how students find or construct relational similarity to design instruction rather than just distinct surface or structure in similarity from his/her point of view and give static tasks to promote transfer (pp. 112-113).

Following the actor-oriented transfer perspective, we changed a classical analogy problem into a more dynamic one by providing learners with opportunity to choose a target object and its property. Let us start with analyzing the task used in Cañadas et al. (2007):

Given a triangle $\mathrm{ABC}$ and a point $\mathrm{P}$ inside the triangle construct the three lines from each vertex A, B, C to the point $\mathrm{P}$. What can you say about the relationships between the lines and the sides of the triangle? (p. 59)

Note that the last part of the task asks students to make a conjecture about the relationships between "some" lines and sides of a triangle without giving any specific objects or relations. To solve the problem, learners need to select "some" lines and look for "a relation", which is similar to what they already know using their language. For example, Cañadas et al. (2007) give the conjecture: "if two lines cut the sides in a 2:1 ratio, the third one will, too" (p. 60).

\footnotetext{
${ }^{1}$ Classical analogy refers to the reasoning that takes the form $\mathrm{A}: \mathrm{B}: \mathrm{C}: \mathrm{D}$, where the $\mathrm{C}$ and $\mathrm{D}$ terms must be related in the same way as the A an B terms are linked. Problem analogy means the analogical reasoning in problem-solving tasks by recognition of similarity between a known problem and a new problem. Pedagogical analogy refers to the reasoning with instructional analogs that are designed to provide a concrete representation of abstract ideas (English, 2004, pp. 4-8).
} 
This conjecture was based on an analogy from knowledge of what happens when lines cut the sides at midpoint. Though the conjecture is false, it can be assumed productive if it is used as a mediation to investigate other new properties of a triangle.

The freedom to create a target object and a relation is not apparent within a classical analogy form because under the condition, the relation between " $\mathrm{A}$ " and " $\mathrm{B}$ " and " $\mathrm{C}$ " are all pre-determined by experts. As a result, there is not much room for students to engage in similarity-making activities that are familiar to them. By leaving the base object's attribute " $\mathrm{B}$ " and the target " $\mathrm{C}$ " and its corresponding property " $\mathrm{D}$ " in a classical analogy to the discretion of a learner, the learner may manage his/her personal journey of knowledge construction based on the learner's own similarity-making action and its results as each participant did in the hypothetical journey of discovery described in Lakatos (1976). Therefore, an analogy that requires learners to search authentically for the "B", "C", and "D" terms of a classical analogy, which we call open classical analogy (hereinafter referred to as OCA), could be used as an instructional device in mathematics classrooms.

\subsection{OCA type problems}

Requiring learners to look for "B", "C", and "D" terms, we can design three types of OCA problems depending which terms are given by teachers and are open for learners' discretion. First, a teacher may opt to start with " $\mathrm{A}$ " and " $\mathrm{C}$ " terms of a classical analogy:

Consider a tetrahedron is similar to a triangle. Conjecture a property of a tetrahedron analogous to the property of a triangle you already know. Explain your answer.

A teacher could also choose to provide students with another two terms, "A" and "B":

The interior angles of a triangle sum to two right angles. Conjecture a similar property of any geometric figure that is analogous to a triangle. Explain your answer.

Finally, it is also possible to start with providing students with only the " $A$ " term:

Select a geometric figure that is analogous to a triangle and make a conjecture about its property that is analogous to a property of a triangle. Explain your answer.

In general, we can modify many problems in school mathematics to OCA type problems. For example, the aforementioned problem given in Cañadas et al. (2007) can be rephrased in the form of OCA problem where " $\mathrm{A}$ " and " $\mathrm{B}$ " terms are given:

Three median lines of a triangle meet in a single point. Select other lines and conjecture a property that is analogous to the given property. Explain your answer.

\subsection{Nature of knowledge construction through OCA problem solving}

Three epistemic actions elaborated in Hershkowitz et al. (2007) such as "recognizing", "building-with", and "constructing" are expected to happen during knowledge construction via OCA problem solving. In the OCA problem-solving process, "recognizing" involves recalling conceptual aspects of a base object and "building-with" consists of combining existing conceptual aspects to select a target or to make a conjectural statement. "Constructing" consists of assembling knowledge artifacts to produce a new structure of a target object as well as a base object. For the case shown in Fig. 1, one should recognize that "median line of a triangle" is related to the special point called "midpoint." Then, one should combine these ideas together and build "a relational concept" such as each median 
Fig. 1 The second type of OCA problem

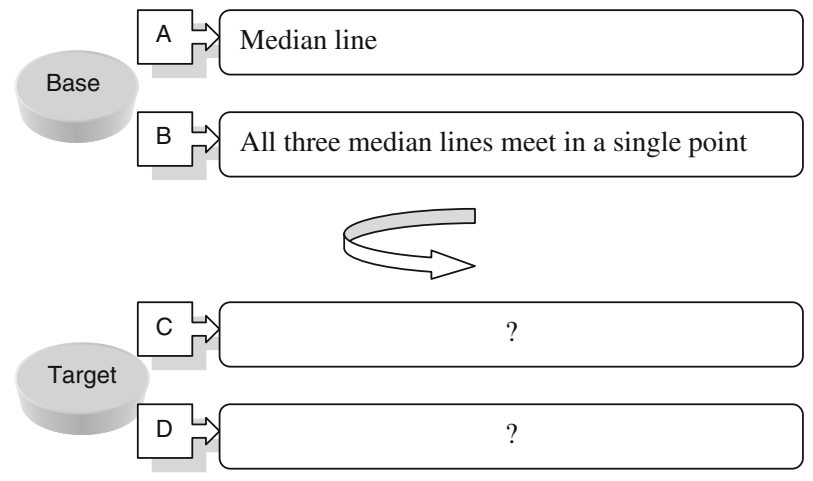

line "divides" one side of a triangle "into two equal parts." The relational concept can be found in another familiar object of a triangle, i.e., angle, then, one can construct a similar statement as a conjecture, i.e., "all three angle bisectors meet in a single point." Thus, conjecturing a similar property of a target object heavily depends on one's building ability of a relational concept of a base object.

For the first and the second types of OCA problems, parts of actions such as finding a property of a base object or looking for an analogous object are not necessary. We hypothesize that there are three different levels of OCA problem solving. The students in the first level might focus only on "the perceptual or surface similarity" of objects as Gentner \& Rattermann (1991) pointed out and use it as a similarity idea for OCA problem solving. Second level of OCA problem solving involves recognition or creation of concept, which we call "transitional similarity," which is relational but not connected to justification context. The highest level of OCA problem solving is supported by "relational similarity", at which the learner can construct new concepts or new properties of a target object in the form of conjectures to be verified.

OCA problems present learners with the chance not only to analyze a base object but also to construct a target object based on recognized or produced similarity. The similarity ideas learners depend on during an OCA problem solving differs from the ideas used in a classical analogy problem solving in two aspects. First, it is a learners' creation not an experts', which prompts learners to employ knowledge they already know. Second, similarity ideas utilized during an OCA problem solving are often provisional similarity ideas, which can be replaced by better ideas or revised. In this case, learners are more involved in identifying or investigating resemblances among geometric objects with their own knowledge. OCA problem usage can also serve as instructional devices to activate learners' prior knowledge and provoke learners to expand their knowledge scope.

Reflective abstraction is facilitated with perspective changing on constructs by analogical reasoning (English \& Sharry, 1996). This "perspective changing" has been expressed as objectification by Dubinsky (1991) or reification by Sfard (1991): objectification or reification of relational similarity. An instructional approach, called "focusing phenomena", employed by Lobato, Ellis and Muñoz (2003), is useful to highlight some mathematical activities to facilitate objectification or reification of relational similarity. As the teacher did in the study by Lobato et al. (2003), "directing students" attention to particular aspects of mathematical activity" (p. 3, emphasis added) would enable students to become aware of a hidden relation and use it while weakening others. More recently, White and Mitchelmore (2010) proposed a teaching for abstraction 
model. This model, which combines post-Piagetian and socio-cultural frameworks, consists of four phases, namely familiarity-similarity-reification-application (FSRA) and extends the Recognizing-Building-Constructing-Consolidation $(\mathrm{RBC}+\mathrm{C})$ model of Hershkowitz et al. (2007) by incorporating contextual rich discourse as integral to the abstraction process.

\section{Method}

\subsection{Participants}

In order to facilitate analogy use for conjecturing in discourse-rich mathematics classrooms, one would expect the experimentation to take place within a mathematics classroom. However, the goal of this study was to identify the types of conjectures that would arise in a more controlled environment, namely outside the regular classroom and with a specific mathematical concept. This was done purposefully to understand first the richness of knowledge construction that students engaged in, as well as to serve as a precursor to studies inside the regular classroom with teachers informed about the findings. Hence, three high-achieving 8th graders (Kim, Park, and Choi, 14 years old) were selected as study participants to investigate the possibility that students can manage knowledge construction during OCA problem solving. Kim, Park, and Choi were selected out of 17 students within the year-long talent development program run by Seoul National University. They had previous exposure to the solving of linguistic analogies and geometric pattern analogies from attempting IQ tests.

One 3-h-long lesson where students were engaged in an OCA problem solving and hourlong clinical interviews both before and after lessons with each student were conducted. The instructor, whom we call Seo, who worked in middle school for 7 years and had an interest in creativity development through student knowledge construction, participated in this research. She followed the teaching perspective that students are able to build meaningful conjectures when challenged to create meaningful analogies through focusing on relational similarities. She had previous experience in such research-oriented activities. She was aware of the goal for OCA problem solving as "seeing the structure of the base object" and "building a structure of a new object (the target)" based on the emergent structure of the base object. For the conceived goal, she encouraged students to "revisit" what they had learned or discovered on the base object and to "project them" to the assumed target object and its property. Her intervention can be characterized as "reflective discourse" (Cobb \& Boufi, 1997, p. 258).

\subsection{The task}

Among the three types of OCA problems, we chose the most open one. In particular, students are given only the "A" term of a classical analogy and required to create the other three terms. The base object for the task was a triangle, learned since students' primary school years.

Select a geometric figure that is analogous to a triangle and make a conjecture about its property that is analogous to a property of a triangle. Explain your answer.

Students are familiar with many aspects of a triangle such as definition, measurement attributes, and properties in the form of mathematical propositions. Thus, students were 
expected to activate their prior knowledge of a triangle to find relational concepts as similarity ideas and make a good conjecture about the selected target object. The task was modified to other types of OCA giving additional information depending on students' reactions. For example, in case students did not use prior knowledge, then, the teacher gave a target object or one specific property (See Section 2.3 for details). Each of the students' answers were evaluated, whether each was complete or not; correct or incorrect, if it had any potential to reveal the hidden structure of a triangle or to create a new structure of their choice for target objects.

\subsection{Data and analysis}

Data about students' previous knowledge of triangle were collected from the one hour-long pre-lesson interviews. Students were asked to explain "what they know about a triangle" including definitions, attributes, and properties. That was to make sure that they had relevant knowledge to construct conjectures of geometrical objects selected to be target objects. Then, students were encouraged to recall "how they have learned about triangle" since primary school. Finally, students were asked to think about "what the process of constructing mathematical knowledge looks like." The famous "eureka" episode of Archimedes was used as a motivating example of analogical reasoning when he found the way to examine if base metal had been substituted for gold in the crown that had been commissioned by his king.

Extensive video-based data was collected on similarity-making activities including processes while solving the given OCA problem. Students were given self-monitoring and self-controlling responsibilities, which is supported and detailed by Zimmerman (1998, 2000 and 2002), while they generated meaningful conjectures. Students were required to consolidate analogy based on their own similarity-making activities. In sum, the OCA task solving for this study was planned according to the following stages: (a) OCA introduction as a thinking format or a discovering tool of mathematics, (b) target object and property searching through their own similarity-recognizing or similarity-making, (c) evaluation and improvement of initial analogies, and (d) consolidation of analogies through justification.

Finally, data about what the participants reflected on in their similarity-making activities and conjecturing were collected from the hour-long post-lesson interview. While watching the recorded activities, students were asked to explain what they "newly" came to know about triangle, geometric objects that they selected as target objects, and properties and so on. In addition, the subjects were asked to explain "how and why" they focused on particular objects or properties. The data from the post-lesson interview were to investigate the possibility for students to be aware of the goal and the use of OCA problem solving in knowledge construction.

Data analysis was done in two ways: by (a) reviewing the hypothesis on similarities that are expected to be made by students to solve OCA tasks and (b) identifying categories of focusing phenomena emerged. We hypothesize that there are three different similarities to be made by students when solving OCA problems: perceptual or surface, transitional, and relational ones (See Section 2.4). We analyzed and coded transcripts of the lesson and the interviews while identifying critical events referred to these three similarities. We identified the critical events or episodes apart from the three similarity categories to test the validity of the hypothesis on similarities, and came to be convinced of it.

Regarding categories of focusing phenomena, data analysis followed the interpretive techniques developed by Glaser and Strauss (1967) and Strauss and Corbin (1990). We 
developed analytic categories of focusing phenomena, coded the transcripts from the lesson, made an agreement on the validity of the categories, and interpreted the categorized critical episodes by using a constant comparative method. We tried to explain how OCA problem solving activities intertwine with abstraction models such as FSRA by White and Mitchelmore (2010) or $\mathrm{RBC}+\mathrm{C}$ by Hershkowitz et al. (2007). A search was conducted for instances in the video data referred to purposeful ways of controlling familiarity and similarity, noticing or recognizing and reifying relational similarity, and consolidating constructs. Three focusing phenomena emerged, including each of the purposes identified in the lesson and the interviews. We passed through the data, finding meanings and making connections across the phenomena. Finally, we came to the three focusing phenomena which support abstraction or knowledge construction via OCA problem solving.

\section{Findings}

Three subjects, Park, Kim, and Choi were identified to have relevant and qualitatively the same prior knowledge about geometric objects such as triangles, polygons, and polyhedrons by the data from pre-lesson interview. One exceptional difference was Park's ability to use formal language when explaining properties of triangle. He used symbols and conventional expressions for proving. Thus, three subjects were assumed to be ready for OCA task solving. However, the overall pictures of their performances were quite different as described in this section. This section is organized into two parts: (a) emerged similarities and conjectures, and (b) reflective discourses to shift student attention while OCA problem solving.

\subsection{Emerged similarities and conjectures}

\subsubsection{Perceptual or surface similarities}

Right from the onset, students leapt directly into the process of looking for perceptually similar figures without reflecting on or careful classification of the properties of a triangle (Gentner \& Rattermann, 1991). For example, one subject, Park, suggested the target could be a figure made by transforming the three sides of a triangle into three curves because the figures would "look similar." Then, Park created target figures by rotating a triangle. The two suggested targets generated through this technique were a conic and another figure made by rotating the triangle - the longest side of the triangle was used as the axis of rotation. Kim and Choi chose a pyramid as the target figure, justifying their choices with the claim that a pyramid contains a triangle as in Kim's comment "I can see triangles in both figures."

As predicted, perceptual similarities were not linked to meaningful conjectures. Students meandered around shapes of some geometric objects not conceptual relations or properties.

\subsubsection{Transitional similarities}

The teacher intended to engage students in focusing phenomena through enlightening students' critical sense of their similarity-making activities. While recalling definitions of geometric figures and concepts about a triangle, students focused on some "common words" in definitions of geometric figures as follows: 
- Quadrilateral is a polygon with four vertices, four sides, and one face

- $\quad$ N-polygon is a polygon with $n$ vertices, $n$ sides, and one face

- Tetrahedron is a polyhedron with four vertices, six sides, and four faces.

Kim claimed "any polygon" could be a potential target object since all polygons contain similar elements - "all are composed of vertices, sides, and faces." Choi, focussed on elements of a triangle, asserted that a tetrahedron is analogous to a triangle since both figures are constructed in accordance with the same principle: a triangle is made by "connecting" one side to a vertex that is not on the side, and a tetrahedron is made by "connecting" one face to a vertex that is not on the face.

Choi, after investigating the number of elements in geometric figures, characterized a triangle as the figure with "the smallest number of vertices, edges, and faces" among plane figures. He concluded a tetrahedron to be an appropriate target object because it has "the smallest number of vertices, edges, and faces among solid figures." Furthermore, he asserted that an arbitrary polyhedron could "decompose into" a tetrahedron just as an arbitrary polygon could "decompose into" a triangle. Noteworthy is his focus on concepts "unrelated" to a triangle that could be used in analogies. Choi explained his analogy between a triangle and a tetrahedron using the idea that both figures "do not have" parallel lines. He also added that the "non-existence" of a diagonal line was common to both a triangle and a tetrahedron.

It was interesting to see students reveal the new aspects of a triangle described as above that they had not focused on before, but the emerged relational concepts such as "nonexistence of diagonal line" or "the smallest number of vertices and edges" were only reconceptualizations of the known object. There were no conjecture generations, so no justification context emerged. Hence, they were classified as transitional similarities.

\subsubsection{Relational similarities}

After a few transitional similarities emerged, the teacher, again, encouraged students to share their target object choices and explain the reasons behind their choices after having analyzed a triangle, polygons in general, and a tetrahedron during the creation of an analogy for the OCA task. Target choices by other students made Park focus on each element in a triangle separately. His investigation led to two separate categorizations of triangles: triangles that could be characterized by the relative lengths of their sides and triangles that could be characterized by the measure of their interior angles (see Fig. 2).

Park attempted a similar classification of quadrilaterals based on the relative lengths of sides and the relative measure of interior angles (see Fig. 3). He claimed the same "classification criteria"; i.e., the relative lengths of sides and measure of interior angles can support similarity between a triangle and a quadrilateral.

Similar classifications of tetrahedrons were attempted by Park and Kim. Park argued that a regular tetrahedron corresponded to an equilateral triangle. He also coined terminology like "right-angled tetrahedron" for a tetrahedron that contained three right-angled triangles

Fig. 2 Park's categorization of triangles

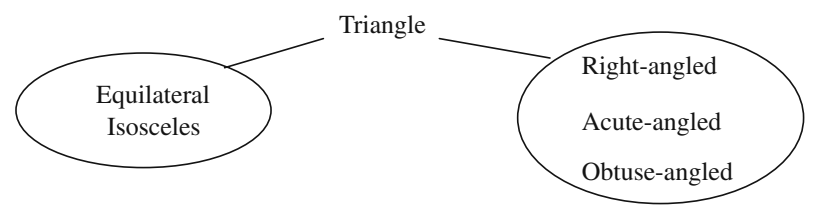




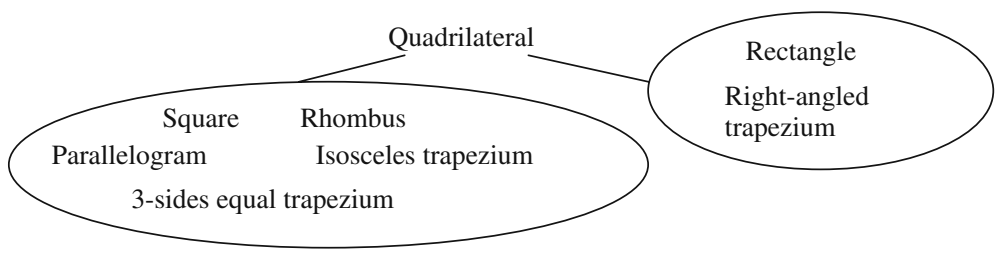

Fig. 3 Park's categorization of quadrilaterals

joined at one vertex and "isosceles tetrahedron" for a tetrahedron that contained three isosceles triangles sides.

The two foci used in the previous classification activities of geometric figures; i.e., side and angle, were analyzed from a different perspective. Kim argued that there were two different kinds of "geometric actions" related to lengths of sides and angle measures. The first was addition or division of the lengths of segments, and the second was addition or division of angle measurements. His three statements, which are very similar in structure, are listed below.

- The interior angles of a triangle always add up to $180^{\circ}$

- The interior angles of a quadrilateral always add up to $360^{\circ}$

- The interior angles of a tetrahedron always add up to "some" degrees

He also constructed the following three similarly structured statements.

- The exterior angles of a triangle always add up to $360^{\circ}$

- The exterior angles of a quadrilateral always add up to $360^{\circ}$

- The exterior angles of a tetrahedron always add up to "some" degrees

The final statements from both analogies are excellent conjectures despite being incomplete. After examining his analogies, Kim pondered the degrees needed to complete his last two statements. Kim and Choi eventually reached a conclusion regarding angle degrees. The two subjects reached quite different conclusions (see Section 4.2.3).

While attempting to generate analogies for the OCA task, Choi focused on area and its measurement in a triangle. He found explanation about the volume measurement of a tetrahedron based on how he learned about the area measurement of a triangle. During the post-task interview, he explained his thought process.

I noticed similarity that I never realized before. I took the idea that triangles between parallel lines with a common base have the same area; then, I analogically determined that tetrahedrons between parallel planes with a common base "must" have the same volume. It was an exciting moment when I found out my analogy was right.

The circumcenter and the incenter of a triangle were used to solve the OCA task, too. Kim, after trying to project these centers on to a quadrilateral easily, found that not all quadrilaterals have those centers. He produced the following statements.

- Three perpendicular side bisectors of a triangle meet at a single point and four perpendicular side bisectors of a square and a rectangle meet at a single point

- Three angle bisectors of a triangle intersect at a single point and four angle bisectors of a square and a rhombus intersect at a single point

Both Kim and Choi tried to generate an analogy between a triangle and a tetrahedron using the existence of two centers. They summarized their work with the statements below. 
- Three perpendicular side bisectors of a triangle meet at a single point and four "unknown" perpendicular bisectors of a tetrahedron meet at a single point

- Three angle bisectors of a triangle intersect at a single point and four "unknown" angle bisectors of a tetrahedron meet at a single point

Kim and Choi independently proceeded to generate an analogy for the OCA task using similar foci. Next is a list of their conjectures regarding the conditions for a quadrilateral and a tetrahedron:

- The lengths of four sides and two angle measures determine a quadrilateral

- If the four sides of a quadrilateral have the same length as the four sides of another quadrilateral and one angle has the same measurement, the two quadrilaterals are congruent

- If four corresponding sides of two quadrilaterals are in proportion and their angles have the same measurement, the two quadrilaterals are similar

- The lengths of six sides determine a tetrahedron

- If the six sides of a tetrahedron have the same length as the six sides in another tetrahedron, the two tetrahedrons are congruent

- If six corresponding sides of two tetrahedrons are in proportion, the two tetrahedrons are similar

\subsection{Focusing phenomena while conjecturing}

Several attention shifts across foci appeared while students made conjectures for the OCA task solving. These attention shifts were made by the teacher or a few students on purpose. In this section, we shall report on the purposes that are the main driving forces of epistemic actions elaborated by Hershkowitz et al. (2007) and cognitive phases elaborated in White and Mitchelmore (2010) while investigating representative cases from critical events or episodes.

\subsubsection{Focusing on the structure and goal of OCA}

Though the teacher, Seo, specifically addressed the necessity of selecting a target, a triangle property, and conjecturing about a target property that corresponded to the selected triangle property, Park could not comprehend the task at hand. The teacher invited students to consider the structure of the OCA after Park selected a target figure created by transforming the three sides of a triangle into three curves based on perceptual similarity. The reflective discourse in a sense that Cobb and Boufi (1997) elaborated between Seo and Park is given below.

45. Park: A triangle has three sides. (Pointing to his drawing of a triangle on the blackboard) I transformed this to this. (Pointing to his drawing of a figure with three curves)

46. Seo: Park, what is your analogy, then?

47. Park: It is this figure.

48. Seo: What analogy are you making with this figure? Can you explain your analogy using your solution?

49. Park: I mean, this figure is similar to a triangle.

50. Seo: Can you explain why it is similar to a triangle?

51. Park: Because I can see shape similarity.

52. Seo: So, how would you write out your analogy? 
53. Park: Wait a minute, I only have the $\mathrm{A}$ and $\mathrm{C}$ terms.

54. Seo: What else do you need?

55. Park: The "B" and "D" terms.

56. Seo: What would "B" be here?

57. Park: It has to be a property of a triangle.

Seo encouraged students to reflect and share emerged relations between a triangle and assumed targets to facilitate students to be aware of the spirit or goal of OCA as "knowledge expansion or extrapolation by conjecturing." After examining his initial constructs, Kim stated, "pondering figures analogous to a triangle, I learnt I need to pay greater attention to good mathematical properties. There must be a good property that can bind a triangle to the new figure and, at the same time, simultaneously distinguish it." Choi assessed his initial target objects as being too superficial, and as a result, deemed them useless. Emerged and potential similarities and found in this vignette.

121. Seo: On what basis did you evaluate your construct and analogy? Would you mind sharing your criteria for analogy evaluation?

122. Kim: The target should be interesting and meaningful and new.

123. Seo: What do you mean by that?

124. Kim: My initial figure, the one I created by rotation, was neither interesting nor meaningful because I couldn't find anything related to the properties of a triangle. I mean, I should have been able to use something I already knew about a triangle.

125. Choi: I had similar thoughts. If I do nothing to generate a new object, I can't encounter anything interesting.

\subsubsection{Focusing on relations originated from geometric actions}

Being directed to reflect on their initial similarity-making activities by the teacher, Kim and Choi became aware of the necessity to recognize relational concepts (similarities) necessary for conjecturing. In other words, students shifted their main attention from the whole image (virtual and conceptual) of a base object to the conceptual elements and the relations between them originated from geometric actions such as "finding minimal conditions" to judge determinance, congruence, and similarity. This attention shift is essential to move to "consolidation" in Hershkowitz et al. (2007) terms, or "reification" and "application" in White and Mitchelmore's (2010). Below is a part of Kim's comments.

The conditions for congruence (of a triangle) are SSS, SAS, and ASA, so I considered the conditions for congruence in other geometric figures. There must be conditions for congruence, for instance, SSSS can definitely work. Hmm, no, it might be SASSS. No, it still doesn't work. It is quite different from a triangle. What if I change a quadrilateral to a parallelogram? Wait a minute. SAS is a condition for congruence. Yes, I can find all the counterparts for the conditions for congruence of a triangle, but not for a scalene quadrilateral.

He also maintained that if the lengths of six sides are given, a tetrahedron is determined. Noteworthy is Kim's word choice. He often used expressions such as "definitely", "doesn't work", "for the case of", "if", "explain", "can find all the counterparts", and so on, which are related to justification of created mathematical conjectures. Likewise, Choi also developed similar vocabulary showing the occurrence of spontaneous interaction between conjecturing and justifying. Hence, it is clear that Kim and Choi were consciously aware of 
the mechanism to find or make relations in geometry focusing on special actions and the necessity of mathematically justifying them. This shows OCA task solving promotes students to develop meta-skills for abstraction through purposeful attention shifting to relational similarity itself.

\subsubsection{Focusing on corresponding}

Building appropriate correspondence between the elements in a base and a target object to complete analogy is one of the essential abilities to complete analogy (English, 2004). There was an interesting discussion on corresponding issue when Kim and Choi made a conjecture about the sum of interior angles in a tetrahedron:

498. Seo: Would you please share what you've discovered?

(Kim and Choi come to the blackboard and write their findings for all to see)

Table 3

Conjectures by Kim and Choi

\begin{tabular}{c|c}
\hline Kim & Choi \\
\hline $\begin{array}{c}\text { The sum of the interior angles of a } \\
\text { tetrahedron is 720 degrees }\end{array}$ & $\begin{array}{c}\text { The sum of the interior angles of a } \\
\text { tetrahedron is constant }\end{array}$ \\
\hline
\end{tabular}

499. Seo: How interesting! Look everyone, Kim and Choi have just written two different conjectures. Let's invite Kim and Choi to enlighten us on the reasons for their conjectures? Who'd like to go first?

500. Kim: A tetrahedron always has four faces. The faces are all triangles. The sum of the interior angles of a triangle is $180^{\circ}$, and there are four triangles. Therefore, the sum of the interior angles of a tetrahedron is $720^{\circ}$.

501. Seo: Any questions for Kim?

502. Choi: I have a question. How do you know the angles you are talking about are indeed the interior angles of a tetrahedron?

503. Kim: (Thinking it over)

504. Seo: Choi, would you mind elaborating? Why are you asking that question?

505. Choi: I mean, there is only one kind of angle in a triangle, but, but, there are, as I found, three different kinds of angles in a tetrahedron. I think we have to decide which angle is the counterpart for the interior angle of a triangle before we make an analogy.

506. Seo: Yes, I see. That's an interesting point. Does anyone want to respond to Choi's comment?

507. Choi: Among the three differing types of angles, the angle composed of three faces at each vertex is the one we're looking for, I think, because the interior angle of a triangle was defined as the angle composed of two sides at each vertex.

508. Kim: I agree, but how do we measure that angle? It seems impossible.

509. Choi: There must be a way; I can't explain it right now.

510. Seo: Then, can you explain your claim that it is constant?

511. Choi: (After drawing a triangle inscribed in a circle) If you draw a triangle in a circle (pointing to his drawing of a triangle, especially the points of the triangle that touch the rim of the circle), the sum of the inscribed angle equals half of the central angle. That's half of 360 or 180 , so the sum of the interior angles of a triangle is always 180, which never changes, I mean, stays constant. (Draws a tetrahedron inscribed in a sphere) Also, if you measure this angle, (points to the angle formed 
from the three faces of the vertex) this angle can be considered an inscribed angle, so it's half of the central angle even though I don't know the actual degree of the central angle. Hence, I can analogically say the sum of these angles is constant just like the triangle case.

Justification vocabulary and ideas used in the above discourse relate to students' corresponding conjectures. Kim's explanation for why the sum of the interior angles is $720^{\circ}$ focused on justification of the conjecture itself (line 500). He concerned himself with verifying the reason the sum totals $720^{\circ}$ based on the fact that all tetrahedral are composed of four triangles and the angle sum of each triangle is $180^{\circ}$. Choi questioned the analogy justification employed by Kim. For Choi, the angles that Kim measured did not correspond to the angles of a triangle, which totaled $180^{\circ}$. He proclaimed it necessary to define a 3-dimensional interior angle that corresponds to a 2-dimensional interior angle. At the post-lesson interview, Kim and Choi made references to the dihedral angle sum though they could not use it to complete the justifications for their conjectures and analogies. This movement from a base to a target with sound and meaningful corresponding concepts appeared to be a useful means of knowledge construction while OCA problem solving.

\section{Discussion and conclusions}

The central theme of the paper concerned students' conjecturing via OCA problem solving as knowledge construction. The detailed analysis of the constructs and processes of several similarity-making and conjecturing activities supported the following conclusions.

First, relational similarity between objects is crucial to be noticed in abstraction (Hershkowitz et al., 2007; White \& Mitchelmore, 2010) and can be recognized by activating learners' use of their own prior knowledge through intentional focusing while OCA problem solving. In this study, employing the reconceptualized format or the structure of a classical analogy was effective to facilitate students' attention shift from surface similarity to transitional and relational similarity. As described in Section 4.1.1, the subjects tended to look for perceptual similarity without reflecting on or careful analyzing the properties of a triangle in the beginning stage. For example, Park's initial target figure was made by transforming three sides of a triangle into three curves. This selection is based on a kind of "make up" strategy — change a part of the given problem situation without any proper understanding or goal, which was a term coined by Lavy and Bershadsky (2003). Intentional focusing on the structure and the goal of the OCA task facilitated Park to pay attention to the relationships among conceptual elements, not perceptual overall features of a base object.

While paying attention to relationships between elements, students investigated the meaning or the results of specific geometric actions such as "dividing sides or angles into equal parts", "classifying geometric objects", "finding angle measures sum", "investigating invariant attributes such as area of triangles between parallel lines", "looking for minimal condition to judge determinance, congruency or similarity of geometric figures", "deciding on object existence or generality", and so on. All the relations students found or created came from the awareness of these geometric actions. For example, Kim noticed that the interior angle measures of a triangle "add up" to $180^{\circ}$ as described in Section 4.1.3. He, then, mapped this to a tetrahedron, and made a statement: the interior angles of a tetrahedron "add up to some" degrees. This statement provoked him to pursue the exact 
degrees as well as its justification as discussed in Section 4.2.3. To sum up, OCA problem solving gives a learner opportunity to experience "intentional" recognizing or creating relations while actively reflecting or re-doing geometric actions that he or she already did on a base object.

Second, conjecturing can be linked to knowledge construction only when it is done with student awareness of the necessity and the method of justification as English and Sharry (1996) pointed out. Surface similarity between geometric objects was not linked to meaningful conjecturing activities as mentioned in Section 4.1.1. When students are concerned only with surface similarities, they stuck to finding a target object rather than conjecturing any properties. Transitional similarities such as "non-existence of diagonals" in a triangle and a tetrahedron were quite interesting to see since they are also new relations for learners. However, transitional similarities are only about descriptions not about conjecturing and its validation, either. Relational similarity, on the other hand, led students to conjectures to be tested or justified. For Kim, as detailed in Section 4.1.3, the judgment condition for determination of a quadrilateral was not the exact projection from, so called, the SSS condition of a triangle. Realizing the complicated relationships between the components and the need to verify similarity in relations, he actively analyzed hypothetical situations and necessary conditions. In Section 4.2.3, Choi also was concerned about conjecturing and justification simultaneously, neither just transforming some part of known properties to build a conjecture nor throwing a sentence without any responsibility of its validation. Thus, his conjecture from OCA problem solving could have potential to become a theorem, which can naturally form cognitive unity (Garuti, Boero \& Lemut, 1998).

Third, knowledge structure of a familiar object can be discovered by intentionally alienating it (Sfard, 1991; Dubinsky, 1991) and an OCA task gives an opportunity to be alienated from familiar objects. In fact, the base object in this study, a triangle, is so familiar for learners to think differently, as pointed out by Mariotti and Fischbein (1997). Interestingly, the subjects often investigated properties of a triangle by converse reasoning. For example, although Kim knew the proof for constructing a circumcenter of a triangle, when inferring about the circumcenter of a tetrahedron, as described in Section 4.2.3, he relied on the reasoning about the target object and applied this reasoning to a triangle conversely. Once inferences had been made on his target object, the process was applied to the base object conversely to reflect knowledge he had from a different perspective. Therefore, seeing or constructing the structure of a base object through OCA problem solving can be regarded as a heuristic to view a familiar object from a new perspective or deal with it in a new way.

Fourth, aspiration of innovation is one of the main driving forces of knowledge construction through conjecturing while OCA problem solving. As described in the empirical context section, the class teacher assumed that students could build knowledge via conjecturing by themselves as Lobato and Siebert (2002) and Zimmerman (1998, 2000, and 2002) elaborated if they were fully engaged in OCA problem solving. With this assumption, she directed reflective discourse on the process and the constructs of OCA problem solving in a sense that Cobb and Boufi (1997, p. 258) suggested. This was helpful for Kim and Choi to grasp the goal of OCA solving and to implement meaningful conjecturing as reported in the result section. While pursuing innovation, Kim and Choi kept continuously searching for interesting targets and related properties to explore mathematical meaning. This aspiration for innovation became their evaluation criterion for conjectures. For example, Kim's comment after reflecting on his initial analogy, "I don't like it because it is too similar to the base object," explicitly reveals his pursuit of innovation (See Section 4.2.1 for more details). Including this, commentary such as "I like 
it because it is very new", "I use it because it comes from the most important property", "I like it because it's simple, yet, cool like the known property", and "It looks great since I had never thought about it like that before" reveal personal feeling about the constructs. I would call this "mathematical taste" that was considered a hidden important factor of productivity in mathematics research (Tao, 2007).

However, the teacher's efforts to direct student attention to the goal or the spirit of OCA were relatively not successful for Park. In Park's journey of conjecturing, it was hard to say that he was pursuing innovation though he produced some properties of a quadrilateral and a tetrahedron. Park's relatively poor performance can be interpreted as partly due to his lack of productive mathematical taste. This phenomenon warrants the necessity to develop an alternative way of awakening the purpose or the role of OCA as well as to develop an appropriate way for cultivating student productive mathematical taste.

The limitation of this research resides with the participants. Student participants constitute high-achieving or more advanced learners. The study group was not constructed of differing level achievers.

Reasoning and conjecturing by analogy is a fundamental human trait. One encounters excellent examples of this propensity to "analogize" in ancient Greek philosophy. If an ancient Greek philosopher were asked: why do we create analogies? The answer would simply be to create a framework by which we could better understand the dimensions of human experience (Sriraman, 2005). An important finding of English (2004) was that teachers must understand analogies themselves and know how to use them effectively (and also know which analogies are appropriate and which are not when it comes to their use). They sometimes have to make the relationships explicit for the child. The OCA framework we have developed through reflective discourse practices by the teacher, Seo, illustrates that analogies arising in mathematics are quite different from those arising in a discipline such as the life sciences where spontaneous analogies work well because children have a much larger a priori linguistic base, whereas in mathematics children's pre-existing knowledge base is limited. This necessitates that both practitioners and researchers are sensitive to the major role that the knowledge base plays in the use of analogies for mathematics learning. The present study makes an important contribution for following this line of mathematical thinking initiated by the likes of Newton, Euler, and Polya. Further research is needed on a more typical classroom-type group of students. Another limitation relates to the limited content area. The focus of this paper was a triangle. Further studies involving a variety of OCA problems in different content areas are encouraged to verify the possibility of including OCA in mathematics learning. Finally, it will be necessary to not only identify but also clarify the kinds of norms or teaching interventions essential for effective integration of OCA into mathematics lessons.

Open Access This article is distributed under the terms of the Creative Commons Attribution Noncommercial License which permits any noncommercial use, distribution, and reproduction in any medium, provided the original author(s) and source are credited.

\section{References}

Bassok, M. (2001). Semantic alignments in mathematical word problems. In Gentner, D., Holyoak, K. J., \& Kokinov, B. N. (Eds.) The analogical mind: Perspectives from cognitive science (pp. 401-433). Cambridge, MA: MIT Press. 
Boero, P., Dreyfus, T., Gravemeijer, K., Gray, E., Hershkowitz, R., Schwarz, B., Sierpinska, A., \& Tall, D. (2002). Abstraction: Theories about the emergence of knowledge structures. In A. D. Cockburn \& E. Nardi (Eds.), Proceedings of the 26th annual conference of the International Group for the Psychology of Mathematics Education (Vol. 1) (pp. 113-138). Norwich: PME.

Cañadas, M. C., Deulofeu, J., Figueiras, L., Reid, D., \& Yevdokimov, A. (2007). The conjecturing process: Perspectives in theory and implications in practice. Journal of Teaching and Learning, 5 (1), 55-72.

Cappetta, R. W., \& Zollman, A. (2009). Creating a discourse rich classroom (DRC) on the concept of limits in calculus: Initiating shifts in discourse to promote reflective abstraction. In L. Knott (Ed.), The role of mathematics discourse in producing leaders of discourse (pp. 17-40). Charlotte: Information Age Publishing.

Cobb, P., \& Boufi, A. (1997). Reflective discourse and collective reflection. Journal for Research in Mathematics Education, 28(3), 258-278.

Dubinsky, E. (1991). Reflective abstraction in advanced mathematical thinking. In D. Tall (Ed.), Advanced mathematical thinking (pp. 95-126). Boston: Kluwer.

Dunbar, K. (2001). The analogical paradox: Why analogy is so easy in naturalistic settings, yet so difficult in the psychology laboratory? In D. Gentner, K. J. Holyoak \& B. K. Kokinov (Eds.), The analogical mind: Perspectives from cognitive science (pp. 313-334). Cambridge: The MIT Press.

English, L. D. (Ed.). (1997). Mathematical reasoning: Analogies, metaphors, and images. Mahwah: Lawrence Erlbaum Associates.

English, L. D. (Ed.). (2004). Mathematical and analogical reasoning of young learners. Mahwah: Lawrence Erlbaum Associates.

English, L. D., \& Sharry, P. V. (1996). Analogical reasoning and the development of algebraic abstraction. Educational Studies in Mathematics, 30, 135-157.

Garuti, R., Boero, P., \& Lemut, E. (1998). Cognitive unity of theorems and difficulties of proof. In A. Oliver \& K. Newstead (Eds.), Proceeding of the 22nd Conference of the International Group for the Psychology of Mathematics Education (pp. 345-352). South Africa: Stellenbosch.

Gentner, D., \& Rattermann, M. J. (1991). Language and the career of similarity. In S. A. Gelman \& J. P. Brynes (Eds.), Perspectives on language and thought: Interrelations in development (pp. 225-277). London: Cambridge University Press.

Glaser, B. G., \& Strauss, A. L. (1967). The discovery of grounded theory: Strategies for qualitative research. Hawthorne: Aldine.

Gray, E., \& Tall, D. (1994). Duality, ambiguity and flexibility: A “proceptual” view of simple arithmetic. Journal for Research in Mathematics Education, 25, 116-140.

Hershkowitz, R., Hadas, N., Dreyfus, T., \& Schwarz, B. (2007). Abstracting processes, from individuals' constructing of knowledge to a group's "shared knowledge”. In M. C. Mitchelmore \& P. White (Eds.), Abstraction in mathematics education [special issue], Mathematics Education Research Journal, 19(2), 41-68.

Holyoak, K. J., \& Thagard, P. (1989). Analogical mapping by constraint satisfaction. Cognitive Science, 13, 295-355.

Lakatos, I. (1976). Proofs and refutations: The logic of mathematical discovery. New York: Cambridge University Press.

Lavy, I., \& Bershadsky, I. (2003). Problem posing via "what if not?" strategy in solid geometry - a case study. Journal of Mathematical Behavior, 22(4), 369-387.

Lee, K. H. (2009). Analogical reasoning by the gifted. In M. Tzekaki, M. Kaldrimidou \& H. Sakonidis (Eds.), Proceeding of the 33rd conference of the International Group for the Psychology of Mathematics Education (pp. 505-512). Greece: Thessaloniki.

Leech, R., Mareschal, D., \& Cooper, R. P. (2008). Analogy as relational priming: A developmental and computational perspective on the origins of a complex cognitive skill. The Behavioral and Brain Sciences, 31, 357-378.

Lobato, J., \& Siebert, D. (2002). Quantitative reasoning in a reconceived view of transfer. The Journal of Mathematical Behavior, 21(1), 87-116.

Lobato, J., Ellis, A. B., \& Muñoz, R. (2003). How "focusing phenomena" in the instructional environment afford students' generalizations. Mathematical Thinking and Learning, 5(1), 1-36.

Mariotti, M. A., \& Fischbein, E. (1997). Defining in classroom activities. Educational Studies in Mathematics, 34, 219-248.

Novick, L.R. (1988). Analogical transfer, problem similarity, and expertise. Journal of Experimental Psychology: Learning, Memory and Cognition, 14(3), 510-520.

Polya, G. (1954). Mathematics and plausible reasoning: Volume I. Princeton: Princeton University Press.

Radford, L. (2008). Connecting theories in mathematics education: Challenges and possibilities. ZDM-The International Journal on Mathematics Education, 40(2), 317-327. 
Reed, S., Dempster, A., Ettinger, M. (1985). Usefulness of analogous solutions for solving algebra word problems. Journal of Experimental Psychology: Learning, Memory and Cognition, 11(1), 106-125.

Richland, L. E., Holyoak, K. J., \& Stigler, J. W. (2004). Analogy use in eighth-grade mathematics classrooms. Cognition and Instruction, 22, 37-60.

Sfard, A. (1991). On the dual nature of mathematical conceptions: Reflections on processes and objects as different sides of the same coin. Educational Studies in Mathematics, 22, 1-36.

Sriraman, B. (2004). Gifted ninth graders' notions of proof. Investigating parallels in approaches of mathematically gifted students and professional mathematicians. Journal for the Education of the Gifted, 27(4), 267-292.

Sriraman, B. (2005). Review of L. English (Ed) (2004). Mathematical and analogical reasoning of young learners. ZDM-The International Journal on Mathematics Education, 37(6), 506-508.

Sriraman, B. (2006). An Ode to Imre Lakatos: Bridging the ideal and actual mathematics classrooms. Interchange: A Quarterly Review of Education, 37(1\&2), 155-180.

Strauss, A., \& Corbin, C. (1990). Basics of qualitative research: Grounded theory procedures and techniques. Newbury Park: Sage.

Tao, T. (2007). What is good mathematics? Bulletin of the American Mathematical Society, 44, $623-634$.

White, P., \& Mitchelmore, M. (2010). Teaching for abstraction: A model. Mathematical Thinking and Learning, 12(3), 205-226.

Zaslavsky, O. (2008). Attention to similarities and differences: a fundamental principle for task design and implementation in mathematics education. Topic Study Group 34: Research and development in task design and analysis, ICME 11. Retrieved June 6, 2009, http://tsg.icme11.org/document/get/290.

Zimmerman, B. J. (1998). Academic studying and the development of personal skill: A self-regulatory perspective. Educational Psychologist, 33, 73-86.

Zimmerman, B. J. (2000). Attaining self-regulation: A social cognitive perspective. In M. Boekaerts, P. Pintrich \& M. Zeidner (Eds.), Handbook of self-regulation (pp. 13-39). San Diego: Academic.

Zimmerman, B. J. (2002). Becoming a self-regulated learner: An overview. Theory into Practice, 41(2), 64-70. 